\title{
PRECLINICAL STUDIES AND CLINICAL TRIALS IN DETERMINATION OF SAFETY AND EFFICACY OF MENINGOCOCCAL VACCINES: PRESENT SCENARIO
}

\author{
SUTAPA BISWAS MAJEE ${ }^{1 *}$, SOUPAYAN PAL ${ }^{2}$ \\ ${ }^{1}$ Department of Pharmacy, NSHM Knowledge Campus, Kolkata-Group of Institutions, 124 B L Saha Road, Kolkata, West Bengal, India. \\ ${ }^{2}$ Department of Pharmaceutical Technology, Jadavpur University, Kolkata, West Bengal, India. Email: sutapabiswas2001@yahoo.co.in
}

Received: 15 December 2020, Revised and Accepted: 27 January 2021

\begin{abstract}
Meningitis, a serious communicable inflammatory disease continues to be a worldwide threat especially in sub-Saharan Africa affecting millions of people with high death rates every year. Strains from six serogroups of the principal causative organism Neisseria meningitidis are found to be responsible for the majority of infections. Incidences of antibiotic resistance and efforts to provide mass protection have necessitated the development of meningococcal vaccines since the latter half of the $20^{\text {th }}$ century. Aiming to ostracize meningitis by 2030 , the World Health Organization focuses on vaccination as an important strategy to reach the goal. Due to limited efficacy and stability issues of earlier polysaccharide and protein conjugate vaccines respectively, outer membrane vesicle (OMV) vaccines were developed. Gene manipulations have also led to the development of more efficacious tailor-made OMV vaccines due to over-expression of antigenic outer membrane proteins along with lesser pyrogenicity. Available data from preclinical studies in animal models and clinical trials, on meningococcal vaccine candidates report the strength of immune response measured by serological tests such as enzyme-linked immunosorbent assay and serum bactericidal assay. Post-immunization adverse reactions have been also monitored as a part of safety assessment. The novelty of the present review lies in summarizing the outcomes of the preclinical animal studies and clinical trials conducted on various types of meningococcal vaccines till date and thereby highlighting the paucities in the existing information which can facilitate understanding the present scenario, challenges, and future scope in the field of meningococcal vaccine development.
\end{abstract}

Keywords: Animal studies, Clinical trials, Immune response, Meningitis, Outer membrane protein, Outer membrane vesicle vaccines, Serological tests. (C) 2021 The Authors. Published by Innovare Academic Sciences Pvt Ltd. This is an open access article under the CC BY license (http://creativecommons.org/ licenses/by/4.0/) DOI: http://dx.doi.org/10.22159/ajpcr.2021v14i4.40509. Journal homepage: https://innovareacademics.in/journals/index.php/ajpcr

\section{INTRODUCTION}

Meningitis, an inflammatory disease of the meninges remains to be a serious global public-health challenge with an estimated 1.2 million cases and 1,35,000 deaths each year caused by the major pathogen Neisseria meningitidis (meningococcus), a Gram-negative bacterium. Based on the chemistry of capsular polysaccharides 12 serogroups of $N$. menigitidis have been identified out of which six $(\mathrm{A}, \mathrm{B}, \mathrm{C}, \mathrm{W}, \mathrm{X}$, and $Y$ ) are recognized to cause most of the meningococcal infections. Serogroups B and C are predominant, followed by serogroup A whereas the other three are emerging. Transmission of infection occurs from person to person through droplets of respiratory or throat secretions from carriers. The pediatric population is found to be most vulnerable with the highest attack rates in infants aged 3-12 months [1].

For treatment of meningitis infections, a range of antimicrobials has been prescribed by the European Society of Clinical Microbiology and Infectious Diseases, the Infectious Disease Society of America, and the World Health Organization (WHO) which include oily chloramphenicol injection (3 g single-dose intramuscular [i.m.]) [2], ceftriaxone i.m. or intravenous injection ( $2 \mathrm{~g} /$ day once daily for 5 days) [3], cefotaxime, amoxicillin, and gentamicin along with injectable dexamethasone (0.4 mg/kg every $12 \mathrm{~h}$ for 2 days) [4] for corticosteroid adjuvant therapy. However, mass vaccination to achieve herd immunity is the best rational approach to prevent pandemic situations and bring down the mortality rate especially in conditions of growing antibiotic resistance. From 2013 to 2020, 33 cases of penicillin-resistant N. meningitidis were reported from the United States, including 11 cases in 2019 to 2020 that were resistant to both penicillin and ciprofloxacin [5]. The Global Meningococcal Initiative recommendations advocate the use of conjugate vaccines to interrupt the acquisition of $N$. meningitidis [6] responsible for Invasive Meningococcal Disease that commonly includes meningitis and septicemia. Moreover, the WHO in its strategy “Defeating Meningitis by 2030: A Global Road Map," (2020) puts much stress on vaccination during baseline situation thereby aiming to eradicate meningitis by 2030 [1].
Before clinical trials, extensive preclinical studies on animal models are essential to develop effective and safe vaccines especially ones with wide coverage. The WHO Guidelines on Nonclinical Evaluation of Vaccines followed by numerous National Regulatory Authorities of different countries deal with the necessary preclinical evaluation and highlight the requisite basic principles as well as specific guidelines for particular vaccines [7]. "European Agency for the Evaluation of Medicinal Products (EMEA) Note for Guidance on Preclinical Pharmacological and Toxicological Testing of Vaccines" provides protocols to determine efficacy and safety profiles of vaccine candidates [8].

Different publications [9-13] on meningococcal vaccines discretely put forward the studies and reports on individual vaccines designed so far with lacunae in information evident in several cases. However, no comprehensive work is available which can give a proper roadmap of meningococcal vaccine development from the latter half of the $20^{\text {th }}$ century till date. The present review article provides an overall glimpse of marketed meningococcal vaccines or the ones at preclinical stages of assessment or in clinical trials. Acquired knowledge can be helpful in understanding the progress, current status, challenges, and future scope in the domain of meningococcal vaccine development.

\section{Meningococcal vaccine types}

Exhaustive review of published reports on meningococcal vaccines reveals those which have been developed since 1974, that is, later half of the $20^{\text {th }}$ century against serogroups A, B, C, W, and Y [14]. Various meningococcal proteins such as GNA2091, GNA1030, Neisserial heparin-binding antigen, factor $\mathrm{H}$ binding protein (fHbp), NadA, Opc, Lbp, and PorA. have been exploited as antigenic targets in vaccine preparation [12]. A shift from mono- to multivalent vaccine has widened the protection coverage due to the presence of antigenic materials from more than one serogroup or strain. Before initiating the discussion on the present scenario of meningococcal vaccines, it is essential to understand the basic concepts pertaining to various classes of meningococcal vaccines categorized on the basis of their composition. 


\section{Capsular polysaccharide vaccines}

The capsular polysaccharide of $N$. meningitidis acts as an antigenic material with multiple epitopes and hence can be used as a vaccine candidate [12]. These are the earliest of all meningococcal vaccines. Initial polysaccharide vaccines included those providing protection against serogroups $\mathrm{A}$ and $\mathrm{C}$. However, later the emergence of serogroups $\mathrm{W}$ and $\mathrm{Y}$ as epidemic strains led to the development of trivalent and tetravalent vaccines (Mencevax ${ }^{\circledR}$ by GlaxoSmithKline and Menomune ${ }^{\circledR}$ by Sanofi-Pasteur) as well. However, polysaccharide vaccines are poorly immunogenic in young infants and children $<2$ years old [15], who are at major risk of acquiring the infection. In a study of a tetravalent polysaccharide vaccine in Saudi Arabia involving 423 children under 5 years of age, bactericidal antibody responses to serogroups A and W were observed in about $90 \%$ and $60 \%$ of 2 and 4 -year-old children, respectively, but only in $25 \%$ and $13 \%$ of 6 and 12 months old infants, respectively [16]. These vaccines also fail to induce immunological memory [17] and cannot induce isotype switching [18].

\section{Conjugate vaccines}

The chemical conjugation of capsular polysaccharide to a protein carrier such as diphtheria and tetanus toxoid ensures a T-cell-dependent immune response [15]. Hence, the resulting protein conjugate vaccines are immunogenic in infants and are capable of imparting long-term immunization through induction of immunological memory [15,19]. In general, nasopharyngeal carriage of meningococci is most frequent in young adults, with a prevalence of $\sim 24 \%$ [20] and approaching $100 \%$ in closed or semi-closed environments, such as military recruit camps and residential schools [21]. Since most transmission occurs in the carriage state, reducing carriage is pivotal to effective vaccination strategies and the use of conjugate vaccines is the most preferable strategy [15]. Previously, meningococcal serogroup C conjugate vaccines, using a genetically detoxified diphtheria toxoid (CRM197) as the protein carrier and developed by Chiron and Wyeth, and using tetanus toxoid as the carrier that has been developed by Baxter, have been successfully used for infant immunization at 2, 3, and 4 months of age in U.K. [22]. Tetravalent conjugate vaccines, effective against serogroups A, C, Y, and W-135 such as Menactra ${ }^{\circledR}$ (diphtheria toxoid being the carrier) (Pfizer) and Nimenrix ${ }^{\circledR}$ (Sanofi-Pasteur) are available in the market.

\section{Outer membrane vesicle (OMV) vaccines}

OMVs are spherical lipid bilayer vesicles that are extruded naturally from the outer membrane (lying beneath the capsule) of Gram-negative bacteria. The size of the membrane vesicles ranges from $\sim 20-250 \mathrm{~nm}$ in diameter [23]. OMVs have a multi-immunogenic capacity for they carry a wide spectrum of endogenous antigens, for example, toll-like receptor (TLR) agonists such as outer membrane proteins (OMPs), lipoproteins and lipopolysaccharide (LPS) $[24,25]$ or lipooligosaccharide (LOS) as in the case of $N$. meningitidis. The oligosaccharide part of LOS acts as the antigenic material and so is capable of eliciting immune response along with the OMPs, thus enhancing protection. Moreover, OMVs are generally superior to polysaccharide vaccines in enhancing phagocytic uptake, as their surface molecules can be better recognized by the humoral immunity components [25]. However, there exists a safety issue regarding use of OMVs as vaccines since they are composed of highly pyrogenic LPS (endotoxin) and can provoke excessive secretion of pro-inflammatory cytokines such as IL-1, IL-8, TNF- $\alpha$, and MIP$1 \beta[23]$ in humans and animals. OMV vaccines can be of three types.

\section{Spontaneous OMVs (sOMVs)}

sOMVs are produced naturally from the bacterial outer membrane by budding phenomenon [26]. Although they are highly immunogenic, pyrogenicity due to high endotoxin content limits their use.

\section{Detergent OMVs (dOMVs)}

dOMVs are isolated by detergent extraction. They are highly safe due to their low content of endotoxin content [27], but the immunogenic response is compromised due to the removal of a large number of surface antigenic proteins by detergents $[26,28]$.

\section{Native OMVs (nOMVs)}

nOMVs are extracted by sonication, a detergent-free method [26]; hence, loss of surface antigens is prevented by keeping a satisfactory immunological profile.

Conjugate vaccines are thermolabile and require a storage temperature of $4^{\circ} \mathrm{C}$ or even lower [29]. Furthermore, structural homology between the meningococcal B capsular polysaccharide and human tissue limits the development of any suitable meningococcal B vaccine using capsular polysaccharide as the antigenic material. Any anticapsular antibody might cross-react with host antigen contributing to autoimmune disease. Thus, the focus on meningococcal vaccine development has shifted to subcapsular antigens [12] where OMV vaccine becomes the preferred choice. Moreover, with an aim to increase efficacy and safety, bioengineered newer generation vaccine candidates were developed in course of time. Penta-acylated lipid A (instead of the normal hexaacyl lipid A of LOS) has been produced by inactivation of $m s B$ gene resulting in lesser pyrogenic OMV vaccines [25]. Gene manipulations have led to the development of tailor-made OMV vaccines with superior immunogenic efficiency due to the over-expression of OMPs (fHbp, NadA, Opc, etc.) $[12,26]$. This leads to increased TLR binding which, in turn, amplifies the immune response [24]. Due to such inherent advantages of safety and better efficacy of OMV-based meningococcal vaccines they form the focal point of the present review.

\section{Assessment procedures in the development and optimization of} meningococcal vaccine candidates

Biopharmaceutical assessment

Rational vaccine development demands judicious selection of adjuvants to modulate immunological response [24,30,31]. For example, Freund's adjuvant exhibits depot effect prolonging vaccine release, thus strengthening immune response [24]. Selection of suitable route of administration is necessary to maximize immune response [31]. Parenteral administration is found to be more efficacious than the intranasal route in terms of bactericidal antibody titer, avidity, and booster response [10,32]. Certain vaccine antigens such as meningococcal serogroup A polysaccharide being acid-labile [29] cannot be delivered orally which necessitates the change of administration route or formulation design. Moreover, the role of biopharmaceutical variables such as particle size, selection of polymers, use of carriers such as liposomes and proteasomes as delivery systems may influence uptake by antigen-presenting cells and subsequent translocation to lymph node $[24,30,31]$. In vivo stability of the vaccine candidate should be properly assessed. Distribution studies should be considered in case of new adjuvants or altered route of administration (e.g., oral or nasal) [8]. Optimization of the vaccine formulation can be done by determining the effect of biopharmaceutical variables on vaccine performance.

\section{Serological tests for assessing vaccine imparted immunity}

Efficacy assessment of vaccines involves serological tests dealing with antibody titer values that are indicative of immune response. Apart from sera, antibody levels in lung homogenates and lung washes are also determined for meningococcal vaccines using enzyme-linked immunosorbent assay (ELISA) [10]. Radioactive Antigen Binding Assay and Solid-Phase Radioimmunoassay are also performed to detect and quantify antibodies [33]. Determination of avidity index is essential to evaluate the overall strength of antigen-antibody interactions [32]. Analysis of antisera by Immunodot assay acts as a faster alternative to ELISA [34]. Hemagglutination Inhibition Assay and Radioactive Bactericidal Test help to investigate antibody responses specific for LPS and different OMPs [33]. ED50 and LD50 values are two related important parameters that are determined to judge the extent of efficacy and safety margin of prepared vaccine candidate [33].

As the presence of serum bactericidal activity predicts vaccine efficacy, serum bactericidal assay (SBA) is performed to analyze complementmediated vaccine-induced immune response [35]. Other serological tests that may be employed as indicative of vaccine imparted immunity 
include opsonophagocytosis assay (OPA) to determine opsonin function along with whole blood assay for cytokine level assessment $[36,37]$. However, determination of strain coverage by SBA is complex and hampered by the small volumes of sera that can be obtained in clinical trials, particularly with infants. The commonly used SBA protocol requires $20 \mu \mathrm{L}$ of serum for a single assay against one target strain, so the number of target strains that can be evaluated is limited [38]. In this context utility of a reproducible and faster alternative (surrogate), the meningococcal antigen typing system must be mentioned that employs a combination of sandwich ELISA and molecular typing of PorA [39]. It helps in determination of strain coverage of the vaccine along with the level of antigenic protein expression even with limited volume of serum sample.

\section{Preclinical studies}

Before exposure of human subjects to vaccine candidates, they need to be tested in animal models as a part of preclinical assessment and also for immunogenicity testing. It is difficult to establish ability of vaccine to elicit immunogenic response without administering it to a biological system. Parameters such as seroconversion rates, geometric mean antibody titers [30] are characterized using animal models in vaccine development. Animal studies are crucial in verifying the outcomes of the in silico mathematical tools such as differential equation (DE) modeling, agent-based modeling, and systematic in silico screening approach with different filtering phases $[30,40]$. Animal models are indispensable for assessment of pharmacodynamic endpoints associated with vaccines. Immunogenicity is considered to be the primary pharmacodynamic endpoint in vaccine development as antibody generation on stimulation of the immune system is the intended role [8]. Secondary endpoints in quality determination of a vaccine deal with biological responses not associated with the desired target and generally include fever, pain at injection site, erythema or occasional adverse effects and thus are related to safety issues [8]. The USP rabbit pyrogen test involves the measurement of body temperature to record any fever response after vaccination. Close monitoring of the vaccinated animal is essential to identify manifestations such as inflammation or erythema. Bodyweight changes are also considered as part of safety studies using animal models [41]. Optimum preclinical data so obtained from animal studies are essential for supporting and proceeding to the subsequent clinical trials of the vaccine candidate. In fact, proper animal studies can even help out in planning effective and safe clinical trials. Correlating titer values of animals with that of humans may establish animal models as surrogates for vaccine quality control.

\section{PRECLINICAL STUDIES ON MENINGOCOCCAL VACCINES}

All animal studies are performed in compliance with Good Laboratory Practice using models closely resembling the human immune response system. Gonzalez et al., 2006 [10], explored the effect of route of administration on humoral immune response generated by OMV vaccine from the epidemic strain CU385 (sero-subtype P1.15 under serogroup B) in murine neonates. Higher bactericidal antibody titers were observed in pooled sera of mice immunized by intraperitoneal (i.p.) route than in mice immunized with intranasal OMV vaccine. However, the intranasal route for meningococcal vaccine delivery is preferred as bacterial invasion occurs through the nasal epithelium and also mucosal antibodies (IgA) are known to be produced. Crossreactivity study by Immunodot for the same vaccine candidate showed that antibodies generated after early life immunization (1 week) reacted with meningococcal antigens present in different strains of the sero-subtype P1.15 which was an advantageous outcome eliciting broader coverage. The possible explanation could be the presence of conserved regions within the major OMPs PorA and PorB and/or minor antigens that are widely expressed across various strains of the same sero-subtype. Furthermore, a comparison of avidity indices and antibody titers of immunized neonatal and adult mice proved the benefit of early life immunization. Poor immunity conferred by the intranasal route may be linked with the passage of much of the vaccine dose into the gastrointestinal tract and subsequent degradation in acidic pH. Immunological memory is an essential characteristic of vaccines that intensifies protection elicited by antibody. The previous studies reported that early life immunization resulted in B cell activation and subsequent differentiation into memory B cells leading to a larger bactericidal response. Thus, it is established that neonatal immunization (through i.p. route) with OMV vaccine against serosubtype P1.15 can result in efficient immunological priming, which can serve as an excellent basis for future protection.

Bakke et al. investigated variation in immune response on the basis of route of administration [9]. Intranasal and subcutaneous (s.c.) vaccinations with serogroup B meningococcal OMV were done in murine models (8-10 weeks old). Sera from mice that were primed intranasally showed bactericidal activity at the same level as those from mice primed subcutaneously. Strikingly this animal study explored the effect of two different routes for primary and secondary immunization. In the majority of animals, antibody responses in serum were stronger after intranasal priming and secondary s.c. immunizations than using intranasal route for both priming and secondary immunizations. Thus, switching to a different administration route during secondary immunization could be advantageous and needs to be established for humans through clinical trials.

Meningococcal B vaccine candidates such as nonavalent PorA containing OMV, OMV with overexpressed fHbp, OMV with overexpressed TbpA, Hsf, NspA, and Omp85, and OMV with overexpressed LbpA and LbpB have been reported to produce serum bactericidal antibodies in mice [12]. However, studies of the same in higher animals could not be found which limits the scope of comparative study based on model differences. However, separate protein formulations containing PorA, TbpA, LbpA, LbpB, etc., were noted to produce immune response in rabbit [42]. Inadequate data failing to highlight the outcomes of studies on meningococcal vaccines against all serogroups in various animal models largely restricted a speedy development and delayed approval process to conduct clinical trials in subsequent stages.

\section{CLINICAL TRIALS}

Vaccine trials should be designed following the WHO protocols on clinical evaluation of vaccines [43]. According to Good Clinical Practice (GCP), investigators should follow Standard Operating Procedures for all study activities with audits at each trial site. Logistic support for vaccine transportation with maintenance of cold chain, if required should be available. Study protocol should clearly define the follow-up schedule of subjects with optimum flexibility without hampering any clinical evaluation [44].

Chronological analysis of clinical trials conducted on meningococcal vaccines reveals inadequate data on vaccines against serogroups other than serogroup B. However, based on data collected through extensive literature survey, it is evident that vaccine trials in different countries have been carried out not only on adult volunteers but also focused greatly on the pediatric population. Like preclinical studies on animal models, clinical trials also include the measurement of antibody titers by ELISA or SBA in human subjects. Antibody levels have been noted after primary immunization and administration of subsequent booster doses to check the efficacy of the immunization schedule as well as any enhancement of immune response and development of immunological memory. Common side effects such as fever and swelling were monitored along with the occurrence of any serious adverse outcome such as anaphylaxis, encephalitis, transverse myelitis, and GuillainBarre syndrome [45].

VA-MENINGOC-BC developed by the Finlay Institute in Cuba was given as two doses and tested in a randomized double-blind controlled trial in 106,000 Cuban school students (aged 10-14 years) from 1987 to 1989. Efficacy for prevention of the Cuban strain (B:4:P1.19,15) of meningococcal disease was estimated to be $83 \%$ during 16 months' observation period [8]. However, Sao Paulo studies (1989-1990) on the same vaccine did not yield similar satisfactory results for children 
from 3 to 83 months $[11,46]$. This poor efficacy might be due to the immature immune system of the younger population involved in the Sao Paulo trial. However, results of animal studies reported by Gonzalez et al. show that early life immunization gave rise to stronger immune response in murine model [10]. The reason behind such contrasting outcomes needs to be investigated.

In 1988-1991, the Norwegian Institute of Public Health, Oslo conducted a trial on MenBvac. It was tested employing a two-dose regimen in a double-blinded, randomized, placebo-controlled trial involving about 172,000 students aged 13-14 years. Vaccine efficacy was calculated to be $57 \%$ after 29 months observation period with antibody levels reported to decline after 10 months. OMVs are characterized by a rapid fall of bactericidal antibodies after primary immunization. For a sustained level of protection, a booster dose (third dose given 10 months after the second dose) has been explored in a separate immunogenicity study and was found to be satisfactory. This supports the previous study of the effect of a booster dose as long as 4-5 years after the primary immunization with two doses in 27 Norwegian adults. [11,47-49].

A Phase I clinical trial of multivalent Por A (Class I OMPs) meningococcal serogroup B OMV vaccine on three groups each having ten healthy adult volunteers revealed the immunogenic response after a single parenteral dose to be sero-subtype-specific, that is, PorA specific. ELISA was performed to measure antibody levels and a dose-dependent increase in antibody titer was observed with higher antibody induction in case of vaccine containing $100 \mu \mathrm{g}$ total protein than that with $50 \mu \mathrm{g}$ protein. Antibody binding was confirmed by Western blotting. Bactericidal assay showed seroconversion ( $\geq$ four-fold increase in bactericidal titer) in the majority of the volunteers. Febrile reaction was not reported in any volunteer; however, local inflammation (pain, redness, and swelling) at the injection site was noted [50].

Aase et al. in 2003 administered a serogroup B OMV vaccine to 12 and 10 volunteers by intranasal and i.m. routes, respectively [32]. After secondary immunization, no booster response was observed in the case of intranasal delivery, whereas a strong booster effect was observed in volunteers receiving the vaccine through i.m. route. This information is contrasting to the previously reported animal study of Bakke et al. published in 2001 [9]. Moreover, a steady and significant maturation of avidity were reported after each i.m. vaccine dose which was absent in the case of intranasal vaccination. A unique feature of this trial was the use of both intranasal drop and intranasal spray in an equal number of volunteers. However, there has been no report on the difference in response due to variation in formulation.

New Zealand clinical trial reports on MeNZB vaccine found it to be $73 \%$ effective in a prospective study on a vaccinated population comprising volunteers $<20$ years using statistical modeling. The study showed satisfactory and prolonged effectiveness of the vaccine candidate in children corresponding to a vaccine effectiveness of $85 \%$ for children aged 6 months $-<3$ years. In contrast to the two-dose immunization schedule with 6 weeks interval followed in Cuban and Norwegian trials, the three-dose regimen with a similar dosing interval in New Zealand trial elicited a higher immune response. For infants, trial data suggested that a fourth dose of MeNZB at 10 months of age (4 months after the primary three-dose course) is required to elicit a booster response as it increased the percentage of SBA responders from $48 \%$ (post third dose) to $69 \%$ (post fourth dose). For detecting possible adverse events as a result of MeNZB administration, an "Intensive Vaccines Monitoring Programme" was established, which prospectively collected data electronically on a cohort of children across New Zealand. Among the three safety studies performed one study evaluated the risk of bronchiolitis and another was involved in monitoring of simple febrile seizures following the administration of MeNZB. The third study investigated the cause-effect relationship of the OMV vaccine (under trial) and the increased risk of Henoch-Schonlein purpura. All three studies proved the vaccine to be safe and devoid of any side or adverse effects [11,51-56].
The Meningitis Vaccine Project successfully developed an effective and safe monovalent Group A conjugate vaccine, MenAfriVacTM, manufactured by Serum Institute of India Ltd. that was tested from 2006 to 2009 at multiple trial sites in sub-Saharan African countries among 2700 subjects aged from 2 months to 29 years of age. However, trial outcomes from African setting could not be found which signifies the existing paucity of information in this field [44,57-60].

A Phase I clinical trial of meningococcal serogroup OMV vaccine with over-expressed fHbp variant 1 (fHbp v.1), two PorAs and OpcA (adhesin protein) with aluminum hydroxide adjuvant was carried out in four groups of adult healthy volunteers (each group assigned to one particular dose) receiving 3 i.m. injections at 6 weeks' intervals. ELISA tests revealed geometric mean antibody levels to LOS and fHbp v.1 to be highest in case of highest dose group on day 98. Both LOS and conserved minor protein antigens appeared to be important targets of bactericidal antibodies as seen from the depletion assays on individual high-titer sera. The vaccine under investigation was found to be nonpyrogenic. However, injection site pain was a noted adverse effect that was most intense in the highest dose group and with the first injection. The increase in plasma fibrinogen was also greatest in the highest dose group and was greatest after the first injection. An increase in WBC count was also observed 2 days post-vaccination [13].

Major clinical trials have been reported in the present review and some others are being presented in the following table (Table 1) comparing the efficacy of meningococcal serogroup B vaccine candidates among different age groups (primarily pediatric and teenage population) and also highlighting the benefit of booster dose in eliciting a similar immune response in younger population [12,61-65].

\section{CHALLENGES AND FUTURE SCOPE}

Vaccine responses are reported to become more strain-specific as the age of the vaccinated population decreases. It has been proved for meningococcal dOMV vaccines and has been observed in infants for the Bexsero vaccine (meningococcal B vaccine by GlaxoSmithKline), thus diminishing coverage [37]. Hence, design of suitable vaccine candidates that can effectively immunize infants and bring down chances of infection demands further research. Uria et al. in 2008 published their findings on three serogroup $\mathrm{C}$ meningococcal isolates that resisted killing by bactericidal antibodies induced by meningococcal $\mathrm{C}$ conjugate vaccine. They identified the presence of an insertion sequence IS 1301 as the cause of this resistance [66]. This information necessitates a continuous process of vaccine development as more such mutations leading to the evolution of resistant strains can occur in due course of time. Specific instrumental analytical techniques are yet to be developed for improved detection of immunological memory. For intranasal vaccine, not much is known about the duration of mucosal antibody responses and immunological memory [32]. Further, the intranasal route requires approximately 10 times higher dose when compared to that of injection due to drainage into the gastrointestinal tract [8]. Incorporation of higher dose even cannot fully equalize the

Table 1: Comparison of the efficacy of meningococcal serogroup $B$ vaccine candidates among different age groups

\begin{tabular}{lllll}
\hline Year & Country & $\begin{array}{c}\text { Number } \\
\text { of doses }\end{array}$ & Age group & Efficacy (\%) \\
\hline 1991 & Norway & 2 & 14-16 years & 97 \\
1991 & Norway & 2 and 1 & 13-14 years & 96 \\
1991 & Norway & 3 and 1 & 12-17 years & 93 \\
1992 & Sao Paulo, Brazil & 2 & 4-6 years & 52 \\
1992 & Sao Paulo, Brazil & 2 & 24-47 months & 43 \\
1992 & Sao Paulo, Brazil & 2 & 3-23 months & 13 \\
2005 & New Zealand & 3 & 16-24 months & 75 \\
2005 & New Zealand & 3 & 6-8 months & 74 \\
2005 & New Zealand & 3 & 6-8 weeks & 54 \\
2008 & UK & 3 & 2 months & 63 \\
2008 & UK & 3 & 6-8 months & 96 \\
\hline
\end{tabular}


efficacies of the two routes [10,32]. Hence, improvement of intranasal vaccine formulation is a challenge for the future [32,67], both in terms of reducing the number of infections and carriage as well as increasing patient compliance. The setup and successful conduct of GCP standard vaccine trials across multiple trial sites located in low-resource settings like African countries are challenging [44]. However, clinical trials at these places are essential for they are prone to the meningococcal epidemic. The correlation between vaccination and incidence of serious adverse incidents such as myalgic encephalomyelitis or chronic fatigue syndrome in human subjects (as in the case of those receiving MenBvac) [11] still needs confirmation through several years of postmarketing surveillance as a part of clinical trials. Moreover, performing preclinical studies using humanized animal models having a human immune system can enhance the entire progress rate. Furthermore, future use of pregnant animals and fixing suitable endpoints such as viability, fetal body weight, and morphology in preclinical studies will be helpful in establishing the safety of meningococcal vaccines during pregnancy. Developmental toxicity studies during the pre-and postnatal period are very important to assess any adverse effect on the fetus/ newborn [7]. Food and Drug Administration recommends the use of the International Council for Harmonization (ICH) S5A guidance document entitled "Detection of Toxicity to Reproduction for Medicinal Products" as a general point of reference to design developmental toxicity studies and evaluation of endpoints for vaccine candidates [68]. Furthermore, the ethical framework for addressing questions concerning placebo use in vaccine trials, as developed by the WHO Department of Ethics and Social Determinants should be followed $[69,70]$.

With the evolution of newer vaccine-resistant strains due to mutation, meningococcal vaccine development remains to be a challenge over the past few decades especially when targeting the pediatric population and utilizing the nasal route. Assessment of vaccine efficacy in terms of detection of immunological memory and generation of detailed safety profile based on both short- and long-term assessments are the future targets. Hence, there exists an immense scope of progress in the field of developing meningococcal vaccines through further preclinical studies and clinical trials.

\section{AUTHORS' CONTRIBUTION}

Equal contribution by both the authors.

\section{CONFLICT OF INTEREST}

None.

\section{AUTHORS FUNDING}

Not applicable.

\section{REFERENCES}

1. World Health Organization. WHO Guideline 2020. Defeating Meningitis by 2030: A Global Road Map. Geneva: World Health Organization; 2020.

2. World Health Organization. Control of Epidemic Meningococcal Disease: WHO Practical Guidelines. $2^{\text {nd }}$ ed. Geneva: World Health Organization; 1997. Available from: https:/www.who.int/csr/resources/ publications/meningitis/WHO EMC BAC $98 \quad 3$ EN/en.

3. WHO Guideline 2007. Standardized Treatment of Bacterial Meningitis in Africa in Epidemic and Non Epidemic Situations. Available from: https://www.apps.who.int/iris/bitstream/handle/10665/69475/WHO CDS_EPR_2007.3_eng.pdf? sequence $=1 \&$ isAllowed $=y$.

4. World Health Organization. WHO Report. Antimicrobial and Support Therapy for Bacterial Meningitis in Children. Report of the Meeting of 18-20 June 1997, Geneva, Switzerland: World Health Organization; 1997. Available from: https://www.who.int/csr/resources/publications/ meningitis/whoemcbac982.pdf?ua $=1$.

5. Antibiotic-resistant $N$. meningitidis Emerges in US. Available from: https://www.healio.com/news/pediatrics/20200619/antibioticresistantn-meningitidis-emerges-in-us. [Last accessed on 2020 Jun 19].

6. Bai X, Borrow R, Bukovski S, Caugant DA, Culic D, Delic S, et al. Prevention and control of meningococcal disease: Updates from the global meningococcal initiative in Eastern Europe. J Infect 2019;79:528-41.

7. Annex I of WHO Technical Report Series, No. 927. WHO Guidelines on Nonclinical Evaluation of Vaccines; 2005. Available from: https:// www.who.int/biologicals/publications/trs/areas/vaccines/nonclinical_ evaluation/ANNEX\%201Nonclinical.P31-63.pdf?ua=1.

8. Committee for Proprietary Medicinal Products. Human Medicines Evaluation Unit. The European Agency for the Evaluation of Medicinal Products 1997. Note for Guidance on Preclinical Pharmacological and Toxicological Testing of Vaccines. Available from: https://www. ema.europa.eu/en/documents/scientific-guideline/note-guidancepreclinical-pharmacological-toxicological-testing-vaccines en.pdf.

9. Bakke H, Lie K, Haugen IL, Korsvold GE, Høiby EA, Naess LB, et al. Meningococcal outer membrane vesicle vaccine given intranasally can induce immunological memory and booster responses without evidence of tolerance. Infect Immun 2001;69:5010-15.

10. González S, Caballero E, Soria Y, Cobas K, Granadillo M, Pajón R. Immunization with Neisseria meningitidis outer membrane vesicles prevents bacteremia in neonatal mice. Vaccine 2006;24:1633-43.

11. Holst J, Martin D, Arnold R, Huergo CC, Oster P, Hallahan JO, et al. Properties and clinical performance of vaccines containing outer membrane vesicles from Neisseria meningitidis. Vaccine 2009;27S:B3-12.

12. Sadarangani M, Pollard AJ. Serogroup B meningococcal vaccines-an unfinished story. Lancet Infect Dis 2010;10:112-24.

13. Keiser PB, Biggs-Cicatelli S, Moran EE, Schmiel DH, Pinto VB, Burden RE, et al. A phase 1 study of a meningococcal native outer membrane vesicle vaccine made from a group B strain with deleted lpxL1 and synX, over-expressed factor $\mathrm{H}$ binding protein, two PorAs and stabilized OpcA expression. Vaccine 2011;29:1413-20.

14. Centers for Disease Control and Prevention. Meningococcal disease. In: Epidemiology and Prevention of Vaccine-preventable Diseases. 13 $3^{\text {th }}$ ed. Atlanta, GA: Centers for Disease Control and Prevention; 2015. p. 231-45. Available from: https://www.cdc.gov/VACCINEs/pubs/ pinkbook/downloads/mening.pdf.

15. Meningococcal Disease, World Health Organization. Available from: https://www.who.int/ith/vaccines/meningococcal/en.

16. Girard PM, Preziosi MP, Aguado MT, Kieny MP. A review of vaccine research and development: Meningococcal disease. Vaccine 2006;24:4692-700.

17. Goldblatt D, Borrow R, Miller E. Natural and vaccine-induced immunity and immunologic memory to Neisseria meningitidis serogroup $\mathrm{C}$ in young adults. J Infect Dis 2002;185:397-400.

18. Test ST, Mitsuyoshi J, Connolly CC, Lucas AH. Increased immunogenicity and induction of class switching by conjugation of complement $\mathrm{C} 3 \mathrm{D}$ to pneumococcal serotype 14 capsular polysaccharide. Infect Immun 2001;69:3031-40.

19. Zhang Q, Finn A. Mucosal immunology of vaccines against pathogenic nasopharyngeal bacteria. J Clin Pathol 2004;57:1015-21.

20. Ravenhorst MB, Bijlsma MW, Houten MA, Struben VM, Anderson AS, Eiden J. Meningococcal carriage in Dutch adolescents and young adults; A cross-sectional and longitudinal cohort study. Clin Microbiol Infect 2017;23:573.e1-573.e7.

21. Caugant DA, Maiden MC. Meningococcal carriage and disease-population biology and evolution. Vaccine 2009;27S:B64-70.

22. Burrage M, Robinson A, Borrow A, Andrews N, Southern J, Findlow J. Effect of vaccination with carrier protein on response to meningococcal C conjugate vaccines and value of different immunoassays as predictors of protection. Infect Immun 2002;70:4946-54.

23. Cai W, Kesavan DK, Wan J, Abdelaziz MH, Su Z, Xu H. Bacterial outer membrane vesicles, a potential vaccine candidate in interactions with host cells based. Diagn Pathol 2018;13:1-12.

24. Sanders H, Feavers IM. Adjuvant properties of meningococcal outer membrane vesicles and the use of adjuvants in Neisseria meningitidis protein vaccines. Expert Rev Vaccines 2011;10:323-34.

25. Kim SH, Kim KS, Lee SR, Kim E, Kim MS, Lee EU, et al. Structural modifications of outer membrane vesicles to refine them as vaccine delivery vehicles. Biochim Biophys Acta 2009;1788:2150-9.

26. Qing G, Gong N, Chen X, Chen J, Zhang H, Wang Y, et al. Natural and engineered bacterial outer membrane vesicles. Biophys Rep 2019;5:184-98.

27. Van der Pol L, Stork M, van der Ley P. Outer membrane vesicles as platform vaccine technology. Biotechnol J 2015;10:1689-706.

28. Zariri A, Beskers J, Waterbeemd BV, Hamstra HJ, Bindels TH, Riet EV, et al. Meningococcal outer membrane vesicle composition-dependent activation of the innate immune response. Infect Immun 2016;84:3024-33.

29. Beresford NJ, Martino A, Feavers IM, Corbel MJ, Bai X, Borrow R, et al. Quality, immunogenicity and stability of meningococcal 
serogroup ACWY-CRM 197, DT and TT glycoconjugate vaccines. Vaccine 2017;35:3598-606.

30. Mantilla JD, Troconiz IF, Garrirdo MJ. ADME processes in vaccines and PK/PD approaches for vaccine optimization. In: ADME and Translational Pharmacokinetics/Pharmacodynamics of Therapeutic Proteins: Applications in Drug Discovery and Development. $1^{\text {st }}$ ed., Ch. 23. New York: Wiley; 2016. p. 347-68.

31. Hudu SA, Shinkafi SH, Umar S. An overview of recombinant vaccine technology, adjuvants and vaccine delivery methods. Int J Pharm Pharm Sci 2016;8:19-24.

32. Aase A, Naess LM, Sandin RH, Herstad TK, Oftung F, Holst J, et al. Comparison of functional immune responses in humans after intranasal and intramuscular immunisations with outer membrane vesicle vaccines against group B meningococcal disease. Vaccine 2003;21:2042-51.

33. Zollinger WD, Mandrell RE, Altieri P, Berman S, Lowenthal J, Artenstein MS. Safety and immunogenicity of a Neisseria meningitidis Type 2 protein vaccine in animals and humans. J Infect Dis 1978:137:728-39.

34. Simple/Rapid Tests, World Health Organization. Available from: https://www.who.int/diagnostics laboratory/faq/simple rapid tests/en.

35. Necchi F, Saul A, Rondini S. Development of a high-throughput method to evaluate serum bactericidal activity using bacterial ATP measurement as survival readout. PLoS One 2017;12:e172163.

36. Finney M, Halliwell D, Gorringe AR. Can animal models predict protection provided by meningococcal vaccines? Drug Discov Today Dis Models 2006;3:77-80.

37. Haren SD, Ganapathi L, Bergelson I, Dowling DJ, Banks M, Samuels RC, et al. In vitro cytokine induction by TLR-activating vaccine adjuvants in human blood varies by age and adjuvant. Cytokine 2016;83:99-109.

38. Gorringe AR, Pajon R. Bexero: A multicomponent vaccine for prevention of meningococcal disease. Hum Vaccines Immunother 2012;8:174-83

39. Boccadifuoco G, Brunelli B, Mori E, Agnusdei M, Gianfaldoni C, Giuliani MM. Meningococcal Antigen Typing System (MATS): A tool to estimate global coverage for $4 \mathrm{CMenB}$, a multicomponent meningococcal B vaccine. Methods Mol Biol 2019;1969:205-15.

40. Patnala K, Zaveri K. Screening of putative therapeutic candidates in superbug (Staphylococcus aureus): A systematic in silico approach. Asian J Pharm Clin Res 2016;9:283-91.

41. Momose H, Mizukami T, Ochiai M, Hamaguchi I, Yamaguchi K. A new method for the evaluation of vaccine safety based on comprehensive gene expression analysis. J Biomed Biotechnol 2010;2010:361841.

42. Christodoulides M, Heckels J. Novel approaches to Neisseria meningitidis vaccine design. Pathog Dis 2017:75:1-16.

43. Annex I: WHO Guidelines on Clinical Evaluation of Vaccines: Regulatory Expectations. Available from: https://www.who.int/ biologicals/publications/clinical_guidelines_ecbs_2001.pdf?ua $=1$.

44. Marchetti E, Mazarin-Diop V, Chaumont J, Martellet L, Makadi MF, Viviani $\mathrm{S}$, et al. Conducting vaccine clinical trials in sub-Saharan Africa: Operational challenges and lessons learned from the Meningitis Vaccine Project. Vaccine 2012;30:6859-63.

45. Stratton K, Ford A, Rusch E, Clayton EW. Adverse Effects of Vaccines: Evidence and Causality. Washington, DC: National Academies Press; 2011.

46. de Moraes JC, Perkins BA, Camargo MC, Hidalgo NT, Barbosa HA, Sacchi CT, et al. Protective efficacy of a serogroup B meningococcal vaccine in Sao Paulo, Brazil. Lancet 1992;340:1074-8.

47. Bjune G, Høiby EA, Grønnesby JK, Arnesen Ø, Holst Fredriksen J, Halstensen $\mathrm{A}$, et al. Effect of outer membrane vesicle vaccine against serogroup B meningococcal disease in Norway. Lancet 1991;338:1093-6.

48. Holst J, Feiring B, Fuglesang JE, Høiby EA, Nøkleby H, Aaberge IS, et al. Serum bactericidal activity correlates with the vaccine efficacy of outer membrane vesicle vaccines against Neisseria meningitidis serogroup B disease. Vaccine 2003;21:734-7.

49. Rosenqvist E, Høiby EA, Wedege E, Bryn K, Kolberg J, Klem A, et al. Human antibody responses to meningococcal outer membrane antigens after three doses of the Norwegian group B meningococcal vaccine. Infect Immun 1995:63:4642-52.

50. Peeters CC, van der Voort EM, Schuller M, Kuipers AJ, van der Ley P, Poolman JT. Phase I clinical trial with a hexavalent PorA containing meningococcal outer membrane vesicle vaccine. Vaccine 1996;14:1009-15.
51. Oster P, O'Hallahan J, Aaberge I, Timan S, Ypma E, Martin D. Immunogenicity and safety of a strain-specific MenB OMV vaccine delivered to under 5-year olds in New Zealand. Vaccine 2007;25:3075-9.

52. Galloway Y, Stehr-Green P, McNicholas A, O'Hallahan J. Use of an observational cohort study to estimate the effectiveness of the New Zealand group B meningococcal vaccine in children aged under 5 years. Int J Epidemiol 2009;38:413-8

53. Tatley MV, Kunac DL, McNicholas A, Zhou L, Ballantyne S, Ashton J, et al. The intensive vaccines monitoring programme (IVMP): An electronic system to monitor vaccine safety in New Zealand. Vaccine 2008:26:2746-52.

54. Stehr-Green P, Galloway Y, Kieft C, McNicholas A. The risk of bronchiolitis hospitalisation following administration of a group B meningococcal vaccine in New Zealand. N Z Med J 2007;120:1-4.

55. Stehr-Green P, Radke S, Kieft C, Galloway Y, McNicholas A, Reid S. The risk of simple febrile seizures after immunisation with a new group B meningococcal vaccine, New Zealand. Vaccine 2008;26:739-42.

56. Sexton K, McNicholas A, Galloway Y, Radke S, Kieft C, Stehr-Green P, et al. Henoch-Schönlein purpura and meningococcal B vaccination. Arch Dis Child 2009;94:224-6.

57. LaForce FM, Okwo-Bele JM. Eliminating epidemic group A meningococcal meningitis in Africa through a new vaccine. Health Aff (Millwood) 2011;30:1049-57.

58. Djingarey MH, Barry R, Bonkoungou M, Tiendrebeogo S, Sebgo R, Kandolo D, et al. Effectively introducing a new meningococcal A conjugate vaccine in Africa: The Burkina Faso experience. Vaccine 2012;30:B40-5.

59. Hirve S, Bavdekar A, Pandit A, Juvekar S, Patil M, Preziosi MP, et al. Immunogenicity and safety of a new meningococcal A conjugate vaccine in Indian children aged 2-10 years: A phase II/III double-blind randomized controlled trial. Vaccine 2012;30:6456-60.

60. Sow S, Okoko B, Diallo A, Viviani S, Borrow R, Carlone G, et al. Immunogenicity and safety of a meningococcal A conjugate vaccine in Africans. N Engl J Med 2011;364:2293-304.

61. Bjune G, Høiby EA, Grønnesby JK, Arnesen O, Fredriksen JH, Halstensen A, et al. Effect of outer membrane vesicle vaccine against group B meningococcal disease in Norway. Lancet 1991;338:1093-6.

62. Feiring B, Fuglesang J, Oster P, Naess LM, Helland OS, Tilman S, et al. Persisting immune responses indicating long-term protection after booster dose with meningococcal group B outer membrane vesicle vaccine. Clin Vaccine Immunol 2006;13:790-6.

63. Oster P, Lennon D, O'Hallahan J, Mulholland K, Reid S, Martin D. MeNZB: A safe and highly immunogenic tailor-made vaccine against the New Zealand Neisseria meningitidis serogroup B disease epidemic strain. Vaccine 2005;23:2191-6.

64. Miller E. Safety and Immunogenicity of Novartis Meningococcal Serogroup B Vaccine (MenB Vaccine) after Three Doses Administered in Infancy. Graz, Austria: 26 ${ }^{\text {th }}$ Annual Meeting of the European Society for Paediatric Infectious Diseases; 2008.

65. Snape MD. Immunogenicity and Reactogenicity of a Novel Serogroup B Neisseria meningitidis Vaccine Administered from 6 Months of Age. Rotterdam, Netherlands: $16^{\text {th }}$ International Pathogenic Neisseria Conference; 2008

66. Uria MJ, Zhang Q, Li Y, Chan A, Exley RM, Gollan B, et al. A generic mechanism in Neisseria meningitidis for enhanced resistance against bactericidal antibodies. J Exp Med 2008;205:1423-34.

67. Haneberg B, Holst J. Can nonliving nasal vaccines be made to work? Expert Rev Vaccines 2002;1:227-32.

68. FDA Guideline 2006. Guidance for Industry: Considerations for Developmental Toxicity Studies for Preventive and Therapeutic Vaccines for Infectious Disease Indications. Available from: https:// www.fda.gov/files/vaccines, \%20blood\%20\&\%20biologics/published/ Guidance-for-Industry--Considerations-for-Developmental-ToxicityStudies-for-Preventive-and-Therapeutic-Vaccines-for-InfectiousDisease-Indications.pdf.

69. Rid A, Saxena A, Baqui AH, Bhan A, Bines J, Bouesseau MC, et al. Placebo use in vaccine trials: Recommendations of a WHO expert panel. Vaccine 2014:32:4708-12.

70. World Health Organization. Expert Consultation on the Use of Placebos in Vaccine Trials. Geneva: World Health Organization; 2013. Available from: https://www.apps.who.int/iris/bitstream/ handle/10665/94056/9789241506250_eng.pdf. 